\title{
Product of composition and differentiation operators and closures of weighted Bergman spaces in Bloch type spaces
}

\section{Li-Xu Zhang ${ }^{1 *}$}

"Correspondence:

cmzhanglixu@163.com

${ }^{1}$ Faculty of Information Technology,

Macau University of Science and

Technology, Macau, China

\begin{abstract}
Closures of weighted Bergman spaces in Bloch type spaces are investigated in the paper. Moreover, the boundedness and compactness of the product of composition and differentiation operators from Bloch type spaces to closures of weighted Bergman spaces spaces in Bloch type spaces are characterized.
\end{abstract}

MSC: 30H30; 30H99; 47B33

Keywords: Weighted Bergman space; Bloch type space; Composition operator

\section{Introduction}

Let $\mathbb{D}=\{z:|z|<1\}$ be the open unit disk in the complex plane $\mathbb{C}$ and $H(\mathbb{D})$ be the class of all functions analytic in $\mathbb{D}$. For $0<p<\infty, H^{p}$ denotes the Hardy space, which consists of all functions $f \in H(\mathbb{D})$ for which (see [6])

$$
\|f\|_{H^{p}}^{p}=\sup _{0<r<1} \frac{1}{2 \pi} \int_{0}^{2 \pi}\left|f\left(r e^{i \theta}\right)\right|^{p} d \theta<\infty .
$$

As usual, $H^{\infty}$ denotes the space of all bounded analytic functions in $\mathbb{D}$.

The Bloch space $\mathcal{B}$ is the set of all functions $f \in H(\mathbb{D})$ that satisfies

$$
\|f\|_{\beta}=\sup _{z \in \mathbb{D}}\left(1-|z|^{2}\right)\left|f^{\prime}(z)\right|<\infty .
$$

It is well known that $\mathcal{B}$ is a Banach space if it is equipped with the norm $\|f\|_{\mathcal{B}}=|f(0)|+\|f\|_{\beta}$. Note that $H^{\infty} \subset \mathcal{B}$. The little Bloch space, denoted by $\mathcal{B}_{0}$, is the subspace of $\mathcal{B}$ consisting of all $f \in H(\mathbb{D})$ such that $\lim _{|z| \rightarrow 1}\left(1-|z|^{2}\right)\left|f^{\prime}(z)\right|=0$. It is well known that $\mathcal{B}_{0}$ is the closure of polynomials in $\mathcal{B}$.

Let $0<\alpha<\infty$. Recall that the Bloch type space, denoted by $\mathcal{B}^{\alpha}$, is the space of all functions $f \in H(\mathbb{D})$ satisfying

$$
\|f\|_{\mathcal{B}^{\alpha}}=|f(0)|+\sup _{z \in \mathbb{D}}\left(1-|z|^{2}\right)^{\alpha}\left|f^{\prime}(z)\right|<\infty .
$$

(c) The Author(s) 2019. This article is distributed under the terms of the Creative Commons Attribution 4.0 International License (http://creativecommons.org/licenses/by/4.0/), which permits unrestricted use, distribution, and reproduction in any medium, provided you give appropriate credit to the original author(s) and the source, provide a link to the Creative Commons license, and indicate if changes were made. 
It is easy to see that $\mathcal{B}^{\alpha}$ is a Banach space under the norm $\|\cdot\|_{\mathcal{B}^{\alpha}}$. Let $n$ be a positive integer. It is well known that $\|f\|_{\mathcal{B}^{\alpha}}$ is equivalent to $\|f\|_{\mathcal{B}^{\alpha, n}}$ (see [34, p. 1149]), where

$$
\|f\|_{\mathcal{B}^{\alpha, n}}=|f(0)|+\left|f^{\prime}(0)\right|+\cdots+\left|f^{(n-1)}(0)\right|+\sup _{z \in \mathbb{D}}\left(1-|z|^{2}\right)^{\alpha+n-1}\left|f^{(n)}(z)\right| .
$$

The little Bloch type space $\mathcal{B}_{0}^{\alpha}$ is the subspace of $\mathcal{B}^{\alpha}$ consisting of all $f \in H(\mathbb{D})$ such that

$$
\lim _{|z| \rightarrow 1}\left(1-|z|^{2}\right)^{\alpha}\left|f^{\prime}(z)\right|=0
$$

For $0<p<\infty$ and $s>-1$, the classical weighted Bergman space $A_{s}^{p}$ consists of those $f \in H(\mathbb{D})$ such that

$$
\|f\|_{A_{s}^{p}}=\left((s+1) \int_{\mathbb{D}}|f(z)|^{p}\left(1-|z|^{2}\right)^{s} d A(z)\right)^{1 / p}<\infty .
$$

Suppose that $\omega$ is a positive and integrable function in $\mathbb{D}$. $\omega$ is called a radial weight if $\omega(z)=\omega(|z|)$. Let $\hat{\omega}(r)=\int_{r}^{1} \omega(t) d t$ for $0 \leq r<1$ and $\hat{\omega}(z)=\hat{\omega}(|z|)$ for $z \in \mathbb{D}$, respectively. $\omega$ is called a doubling weight, denoted by $\omega \in \hat{\mathcal{D}}$, if there is a constant $C>0$ such that

$$
\hat{\omega}(r)<C \hat{\omega}\left(\frac{1+r}{2}\right), \quad \text { when } 0 \leq r<1 .
$$

$\omega$ is called a regular weight, denoted by $\omega \in \mathcal{R}$, if there is a constant $C>0$ determined by $\omega$ such that

$$
\frac{1}{C}<\frac{\hat{\omega}(r)}{(1-r) \omega(r)}<C, \quad \text { when } 0 \leq r<1 .
$$

$\omega$ is called a reverse doubling weight, denoted by $\omega \in \check{\mathcal{D}}$, if there exist constants $K=K(\omega)>$ 1 and $C=C(\omega)>1$ such that

$$
\hat{\omega}(r) \geq C \hat{\omega}\left(1-\frac{1-r}{K}\right), \quad 0 \leq r<1 .
$$

We write $\mathcal{D}=\hat{\mathcal{D}} \cap \check{\mathcal{D}}$. Weights in $\mathcal{D}$ are said to be two-sided doubling weights. They originated in the work of Peláez and Rättyä [22, 23].

Let $0<p<\infty$ and $\omega \in \mathcal{D}$. The weighted Bergman space $A_{\omega}^{p}(\mathbb{D})=A_{\omega}^{p}$ is the space of all $f \in H(\mathbb{D})$ for which

$$
\|f\|_{A_{\omega}^{p}}^{p}=\int_{\mathbb{D}}|f(z)|^{p} \omega(z) d A(z)<\infty
$$

It is easy to check that $A_{\omega}^{p}$ is a Banach space when $p \geq 1$ and a complete metric space with the distance $\rho(f, g)=\|f-g\|_{A_{\omega}^{p}}^{p}$ when $0<p<1$. When $\omega(z)=\left(1-|z|^{2}\right)^{s}(s>-1)$, the space $A_{\omega}^{p}$ becomes the classical weighted Bergman space $A_{s}^{p}$.

Let $\varphi$ be an analytic self-map of $\mathbb{D}$. Every $\varphi$ induces a composition operator $C_{\varphi}$, which is defined on $H(\mathbb{D})$ by

$$
C_{\varphi}(f)(z)=f(\varphi(z)), \quad z \in \mathbb{D} .
$$


Composition operators have been widely studied on various Banach spaces of analytic functions in recent years. One of the main themes when studying composition operators is to characterize the operator properties of $C_{\varphi}$ in terms of the function properties of the symbol $\varphi$ when $C_{\varphi}$ acts on several Banach spaces of analytic functions. Readers can refer to [5], which is a standard introductory reference for the theory of composition operators written by Cowen and MacCluer.

It is a well-known consequence of the Schwartz-Pick lemma that the composition operators are bounded on the classical Bloch space. In 1995, Madigan and Matheson [21] characterized continuity and compactness for composition operators on the Bloch space. Another two compactness criteria for composition operators on the Bloch space have been obtained in [28] and [30]. More characterizations of the boundedness and compactness of composition operators between different Bloch type spaces were studied, for instance, in $[12,18,19,32]$ (see also the references therein).

Let $D$ be the differentiation operator $D f=f^{\prime}, f \in H(\mathbb{D})$. It is well known that the differentiation operator is unbounded on various Banach spaces of analytic functions. The products of composition and differentiation operators $D C_{\varphi}$ and $C_{\varphi} D$ are defined by

$$
D C_{\varphi}(f)=(f \circ \varphi)^{\prime}=f^{\prime}(\varphi) \varphi^{\prime} \text { and } C_{\varphi} D(f)=f^{\prime}(\varphi) .
$$

For a nonnegative integer $m \in \mathbb{N}$, we define $D^{m} f=f^{(m)}, f \in H(\mathbb{D})$. It is natural to define the product of composition operator and $m$ th differentiation operator as follows:

$$
C_{\varphi} D^{m} f=f^{(m)} \circ \varphi, \quad f \in H(\mathbb{D}) .
$$

See $[13-17,37]$ for the study of the product of composition and differentiation operators on various spaces of analytic functions.

Let $X$ and $Y$ be two Banach spaces of analytic functions. For simplicity, the closure of $X \cap Y$ in the norm of $Y$ is denoted by $\mathcal{C}_{Y}(X \cap Y)$. The question, which was raised by Anderson, Clunie, and Pommerenke in [2], of describing the closure of $H^{\infty}$ in $\mathcal{B}$ is still open. Anderson in [1] mentioned that Jones gave an unpublished characterization of the closure of BMOA in $\mathcal{B}$. Ghatage and Zheng in [10] provided a complete proof for Jones's description. In 2008, Zhao studied $\mathcal{C}_{\mathcal{B}}(F(p, p-2, s))$ when $1 \leq p<\infty$ and $0<s \leq 1$ in [31]. Aulaskari and Zhao studied composition operators from the Bloch space to $\mathcal{C}_{\mathcal{B}}(F(p, p-2, s))$ in [3]. Monreal Galán and Nicolau in [7] characterized the closure in the Bloch norm of the space $H^{p} \cap \mathcal{B}$ for $1<p<\infty$. Recently, Galanopoulos, Monreal Galán, and Pau in [8] extended the above result to the case of the unit ball in $\mathbb{C}^{n}$ and for $0<p<\infty$. Bao and Göğuss in [4] characterized the closure of $\mathcal{D}_{\alpha}^{2} \cap \mathcal{B}(-1<\alpha \leq 1)$ in the Bloch space, where $\mathcal{D}_{\alpha}^{2}$ is the Dirichlet type space. See $[9,11,20,26,27,29]$ for more results of the closure of some function spaces in the Bloch space.

It is well known that in many aspects the Hardy space $H^{p}$ is the limit of $A_{\alpha}^{p}$ as $\alpha \rightarrow$ -1 . However, this is a rough estimate. From $[22,23]$, we see that $A_{\omega}^{p}$ induced by rapidly increasing weights lie "closer" to $H^{p}$ than any $A_{\alpha}^{p}$, i.e.,

$$
H^{p} \subset A_{\omega}^{p} \subset A_{\alpha}^{p}
$$

In addition, for any $\alpha>-1$ and $0<p<\infty, \mathcal{B} \subset A_{\alpha}^{p}$ and $\mathcal{B} \subsetneq A_{\omega}^{p}$ for some $\omega \in \hat{\mathcal{D}}$. Motivated by the above observations and [7], we naturally look for a characterization of $\mathcal{C}_{\mathcal{B}}\left(A_{\omega}^{p} \cap \mathcal{B}\right)$ 
or, more generally, of $\mathcal{C}_{\mathcal{B}^{\beta}}\left(A_{\omega}^{p} \cap \mathcal{B}^{\beta}\right)$. The purpose of this paper is to study the closure of $A_{\omega}^{p} \cap \mathcal{B}^{\beta}$ in the norm of $\mathcal{B}^{\beta}$. We give a complete characterization for $\mathcal{C}_{\mathcal{B}^{\beta}}\left(A_{\omega}^{p} \cap \mathcal{B}^{\beta}\right)$. Moreover, we study the boundedness and compactness of the operators $C_{\varphi} D^{m}: \mathcal{B}^{\alpha}\left(\mathcal{B}_{0}^{\alpha}\right) \rightarrow$ $\mathcal{C}_{\mathcal{B}^{\beta}}\left(A_{\omega}^{p} \cap \mathcal{B}^{\beta}\right)$ and $C_{\varphi} D^{m}: \mathcal{C}_{\mathcal{B}^{\beta}}\left(A_{\omega}^{p} \cap \mathcal{B}^{\beta}\right) \rightarrow \mathcal{C}_{\mathcal{B}^{\beta}}\left(A_{\omega}^{p} \cap \mathcal{B}^{\beta}\right)$.

Throughout this paper, we say that $f \lesssim h$ if there exists a constant $C>0$ such that $f \leq C h$. The symbol $f \approx h$ means that $f \lesssim h \lesssim f$.

\section{Characterization of $\mathcal{C}_{\mathcal{B}^{\beta}}\left(A_{\omega}^{p} \cap \mathcal{B}^{\beta}\right)$}

To state and prove our main results in this paper, we need some lemmas. The following well-known estimate can be found in [35, Lemma 3.10].

Lemma 1 Suppose $s>0$ and $t>-1$. Then there exists a positive constant $C$ such that

$$
\int_{\mathbb{D}} \frac{\left(1-|w|^{2}\right)^{t}}{|1-\bar{z} w|^{2+t+s}} d A(w) \leq \frac{C}{\left(1-|z|^{2}\right)^{s}}
$$

for all $z \in \mathbb{D}$.

Lemma 2 ([35, Exercise 8, p. 63]) For any $T>0$, there exists a constant $C>0$ (depending on $T$ but not on $t$ and $z)$ such that

$$
\int_{0}^{2 \pi} \frac{d \theta}{\left|1-z e^{i \theta}\right|^{1+t}} \leq \frac{C \Gamma(t)}{(1-|z|)^{t}}
$$

for all $z \in \mathbb{D}$ and $t \in(0, T)$. Here $\Gamma(t)=\int_{0}^{+\infty} x^{t-1} e^{-x} d x$.

Lemma 3 ([25, Proposition 5]) Let $0<p<\infty$ and $\omega \in \hat{\mathcal{D}}$, and write $W(r)=W_{\omega}(r)=\frac{\hat{\omega}(r)}{1-r}$ for all $0 \leq r<1$. Then $\|f\|_{A_{W}^{p}} \approx\|f\|_{A_{\omega}^{p}}$ for all $f \in H(\mathbb{D})$ if and only if $\omega \in \mathcal{D}$. Moreover, if $\omega \in \mathcal{D}$, we have $W \in \mathcal{R}$ and $\hat{W}=\hat{\omega}$.

Lemma 4 ([24, Theorem 5]) Let $\omega \in \hat{\mathcal{D}}, 0<p<\infty$, and $k \in \mathbb{N}$. Then

$$
\|f\|_{A_{\omega}^{p}}^{p} \approx \int_{\mathbb{D}}\left|f^{(k)}(z)\right|^{p}(1-|z|)^{k p} \omega(z) d A(z)+\sum_{j=0}^{k-1}\left|f^{(j)}(0)\right|^{p}, \quad f \in H(\mathbb{D})
$$

if and only if $\omega \in \mathcal{D}$.

Now we are in a position to state and prove our main results in this paper.

Theorem 1 Let $n$ be a positive integer. Suppose that $0<p<\infty, \omega \in \mathcal{D}$, and $0<\beta<\infty$. Let $f \in \mathcal{B}^{\beta}$. Then $f \in \mathcal{C}_{\mathcal{B}^{\beta}}\left(A_{\omega}^{p} \cap \mathcal{B}^{\beta}\right)$ if and only if, for any $\epsilon>0$,

$$
\int_{\Omega_{n, \beta, \epsilon}(f)} \frac{\hat{\omega}(z) d A(z)}{\left(1-|z|^{2}\right)^{p \beta-p+1}}<\infty
$$

where

$$
\Omega_{n, \beta, \epsilon}(f)=\left\{z \in \mathbb{D}:\left(1-|z|^{2}\right)^{\beta+n-1}\left|f^{(n)}(z)\right| \geq \epsilon\right\} .
$$


Proof Let $W(z)=\frac{\hat{\omega}(z)}{1-|z|^{2}}=\frac{\hat{\omega}(|z|)}{1-|z|^{2}}$. By Lemma 3, $\|f\|_{A_{W}^{p}} \approx\|f\|_{A_{\omega}^{p}}$. Hence,

$$
f \in \mathcal{C}_{\mathcal{B}^{\beta}}\left(A_{W}^{p} \cap \mathcal{B}^{\beta}\right) \quad \Leftrightarrow \quad f \in \mathcal{C}_{\mathcal{B}^{\beta}}\left(A_{\omega}^{p} \cap \mathcal{B}^{\beta}\right)
$$

Take a function $f$ in the closure in the Bloch type norm of $A_{W}^{p} \cap \mathcal{B}^{\beta}$ and $\epsilon>0$. Then there exists $g \in A_{W}^{p} \cap \mathcal{B}^{\beta}$ such that $\|f-g\|_{\mathcal{B}^{\beta, n}} \leq \frac{\epsilon}{2}$. Note that

$$
\begin{aligned}
\left(1-|z|^{2}\right)^{\beta+n-1}\left|f^{(n)}(z)\right| \leq & \sup _{w \in \mathbb{D}}\left(1-|w|^{2}\right)^{\beta+n-1}\left|f^{(n)}(w)-g^{(n)}(w)\right| \\
& +\left(1-|z|^{2}\right)^{\beta+n-1}\left|g^{(n)}(z)\right| \\
\leq & \frac{\epsilon}{2}+\left(1-|z|^{2}\right)^{\beta+n-1}\left|g^{(n)}(z)\right|, \quad z \in \mathbb{D} .
\end{aligned}
$$

This implies that $\Omega_{n, \beta, \epsilon}(f) \subseteq \Omega_{n, \beta, \frac{\epsilon}{2}}(g)$. Then, by Lemmas 3 and 4 , we have

$$
\begin{aligned}
\|g\|_{A_{\omega}^{p}}^{p} & \geq \int_{\mathbb{D}}\left|g^{(n)}(z)\right|^{p}\left(1-|z|^{2}\right)^{n p} W(z) d A(z) \\
& \geq \int_{\Omega_{n, \beta, \frac{\epsilon}{2}}(g)}\left|g^{(n)}(z)\right|^{p}\left(1-|z|^{2}\right)^{n p} W(z) d A(z) \\
& =\int_{\Omega_{n, \beta, \frac{\epsilon}{2}}(g)} \frac{\left|g^{(n)}(z)\right|^{p}\left(1-|z|^{2}\right)^{(\beta+n-1) p} W(z)}{\left(1-|z|^{2}\right)^{(\beta+n-1) p-n p}} d A(z) \\
& \geq\left(\frac{\epsilon}{2}\right)^{p} \int_{\Omega_{n, \beta, \frac{\epsilon}{2}}(g)} \frac{W(z) d A(z)}{\left(1-|z|^{2}\right)^{p \beta-p}} \\
& \geq\left(\frac{\epsilon}{2}\right)^{p} \int_{\Omega_{n, \beta, \epsilon}(f)} \frac{W(z) d A(z)}{\left(1-|z|^{2}\right)^{p \beta-p}},
\end{aligned}
$$

which implies that

$$
\int_{\Omega_{n, \beta, \epsilon}(f)} \frac{\hat{\omega}(z) d A(z)}{\left(1-|z|^{2}\right)^{p \beta-p+1}}=\int_{\Omega_{n, \beta, \epsilon}(f)} \frac{W(z) d A(z)}{\left(1-|z|^{2}\right)^{p \beta-p}}<\infty .
$$

Conversely, assume that (1) holds. Fix $\epsilon>0$ and let $f$ satisfy (1). Without loss of generality, we may assume that $f(0)=f^{\prime}(0)=\cdots=f^{(n-1)}(0)=0$. For any $z \in \mathbb{D}$, using Proposition 4.27 in [35], we have

$$
f(z)=\frac{1}{(\gamma+2) \cdots(\gamma+n)} \int_{\mathbb{D}} \frac{f^{(n)}(w)\left(1-|w|^{2}\right)^{n+\gamma}}{(1-z \bar{w})^{2+\gamma} \bar{w}^{n}} d A(w),
$$

where $\gamma \geq 0$. Then we will choose $\gamma$ large enough for our purpose. Following [31], we set $f(z)=f_{1}(z)+f_{2}(z)$, where

$$
f_{1}(z)=\frac{1}{(\gamma+2) \cdots(\gamma+n)} \int_{\Omega_{n, \beta, \epsilon}(f)} \frac{f^{(n)}(w)\left(1-|w|^{2}\right)^{n+\gamma}}{(1-z \bar{w})^{2+\gamma} \bar{w}^{n}} d A(w)
$$

and

$$
f_{2}(z)=\frac{1}{(\gamma+2) \cdots(\gamma+n)} \int_{\mathbb{D} \backslash \Omega_{n, \beta, \epsilon}(f)} \frac{f^{(n)}(w)\left(1-|w|^{2}\right)^{n+\gamma}}{(1-z \bar{w})^{2+\gamma} \bar{w}^{n}} d A(w) .
$$


Obviously,

$$
f_{1}^{(n)}(z)=(\gamma+n+1) \int_{\Omega_{n, \beta, \epsilon}(f)} \frac{f^{(n)}(w)\left(1-|w|^{2}\right)^{n+\gamma}}{(1-z \bar{w})^{n+2+\gamma}} d A(w)
$$

and

$$
f_{2}^{(n)}(z)=(\gamma+n+1) \int_{\mathbb{D} \backslash \Omega_{n, \beta, \epsilon}(f)} \frac{f^{(n)}(w)\left(1-|w|^{2}\right)^{n+\gamma}}{(1-z \bar{w})^{n+2+\gamma}} d A(w) .
$$

Let $h(z)=f_{1}(z)-\sum_{k=1}^{n-1} \frac{f_{1}^{(k)}(0)}{k !} z^{k}$. Then $h(0)=h^{\prime}(0)=\cdots=h^{(n-1)}(0)=0$ and $(f-h)^{(n)}(z)=$ $f_{2}^{(n)}(z)$. Using Lemma 1 , we obtain

$$
\begin{aligned}
\|f-h\|_{\mathcal{B}, n}, n & =\sup _{z \in \mathbb{D}}\left(1-|z|^{2}\right)^{\beta+n-1}\left|f_{2}^{(n)}(z)\right| \\
& \lesssim \sup _{z \in \mathbb{D}}\left(1-|z|^{2}\right)^{\beta+n-1} \int_{\mathbb{D} \backslash \Omega_{n, \beta, \epsilon}(f)} \frac{\left|f^{(n)}(w)\right|\left(1-|w|^{2}\right)^{n+\gamma}}{|1-z \bar{w}|^{n+2+\gamma}} d A(w) \\
& \lesssim \epsilon \sup _{z \in \mathbb{D}}\left(1-|z|^{2}\right)^{\beta+n-1} \int_{\mathbb{D}} \frac{\left(1-|w|^{2}\right)^{\gamma-\beta+1}}{|1-z \bar{w}|^{n+2+\gamma}} d A(w) \\
& \lesssim \epsilon .
\end{aligned}
$$

This means that $h \in \mathcal{B}^{\beta}$.

Since $W \in \mathcal{R}$, it is well known that, see [22] for example, there exist $-1<a<b<\infty$ and $\delta \in[0,1)$ such that

$$
\frac{W(r)}{(1-r)^{b}} \nearrow \infty, \quad \text { and } \quad \frac{W(r)}{(1-r)^{a}} \searrow 0, \quad \text { when } r \geq \delta .
$$

Without loss of generality, we can assume $\delta=0$. Then we can choose $\gamma>0$ such that $(p-1)(\beta-1)+\gamma-|b|>0$. By Lemmas 1 and 2 , we have

$$
\begin{aligned}
& \int_{|z| \leq|w|} \frac{\left(1-|z|^{2}\right)^{n-(p-1)(\beta-1)} W(z)}{|1-z \bar{w}|^{n+2+\gamma}} d A(z) \\
& \approx \int_{0}^{|w|}\left(1-r^{2}\right)^{n-(p-1)(\beta-1)} W(r) d r \int_{0}^{2 \pi} \frac{1}{\left|1-r \bar{w} e^{i \theta}\right|^{n+2+\gamma}} d \theta \\
& \quad \approx \int_{0}^{|w|} \frac{W(r)}{(1-r)^{b}} \frac{(1-r)^{n-(p-1)(\beta-1)+b}}{(1-r|w|)^{n+\gamma+1}} d r \\
& \lesssim \frac{W(w)}{(1-|w|)^{b}} \int_{0}^{|w|} \frac{1}{(1-r)^{(p-1)(\beta-1)+\gamma-b+1}} d r \\
& \lesssim \frac{W(w)}{(1-|w|)^{(p-1)(\beta-1)+\gamma}}
\end{aligned}
$$

and

$$
\begin{aligned}
& \int_{|w|<|z|<1} \frac{\left(1-|z|^{2}\right)^{n-(p-1)(\beta-1)} W(z)}{|1-z \bar{w}|^{n+2+\gamma}} d A(z) \\
& \lesssim \hat{\omega}(w) \int_{|w|<|z|<1} \frac{\left(1-|z|^{2}\right)^{n-(p-1)(\beta-1)-1}}{|1-z \bar{w}|^{n+2+\gamma}} d A(z)
\end{aligned}
$$




$$
\begin{aligned}
& \leq \hat{\omega}(w) \int_{\mathbb{D}} \frac{\left(1-|z|^{2}\right)^{n-(p-1)(\beta-1)-1}}{|1-z \bar{w}|^{n+2+\gamma}} d A(z) \\
& \lesssim \frac{W(w)}{(1-|w|)^{(p-1)(\beta-1)+\gamma}} .
\end{aligned}
$$

Thus using Fubini's theorem and Lemma 1, we obtain

$$
\begin{aligned}
& \int_{\mathbb{D}}\left|h^{(n)}(z)\right|^{p}\left(1-|z|^{2}\right)^{n p} W(z) d A(z) \\
& =\int_{\mathbb{D}}\left|f_{1}^{(n)}(z)\right|^{p}\left(1-|z|^{2}\right)^{n p} W(z) d A(z) \\
& \leq\left\|f_{1}\right\|_{\mathcal{B}^{\beta, n}}^{p-1} \int_{\mathbb{D}}\left|f_{1}^{(n)}(z)\right|\left(1-|z|^{2}\right)^{n-(p-1)(\beta-1)} W(z) d A(z) \\
& \lesssim\left\|f_{1}\right\|_{\mathcal{B}^{\beta, n}}^{p-1} \int_{\mathbb{D}}\left(1-|z|^{2}\right)^{n-(p-1)(\beta-1)} W(z) \\
& \times\left(\int_{\Omega_{n, \beta, \epsilon}(f)} \frac{\left|f^{(n)}(w)\right|\left(1-|w|^{2}\right)^{n+\gamma}}{|1-z \bar{w}|^{n+2+\gamma}} d A(w)\right) d A(z) \\
& \lesssim\left\|f_{1}\right\|_{\mathcal{B}^{\beta, n}}^{p-1} \int_{\Omega_{n, \beta, \epsilon}(f)}\left|f^{(n)}(w)\right|\left(1-|w|^{2}\right)^{n+\gamma} \\
& \times\left(\int_{\mathbb{D}} \frac{\left(1-|z|^{2}\right)^{n-(p-1)(\beta-1)} W(z)}{|1-z \bar{w}|^{n+2+\gamma}} d A(z)\right) d A(w) \\
& \lesssim\left\|f_{1}\right\|_{\mathcal{B}^{\beta, n}}^{p-1} \int_{\Omega_{n, \beta, \epsilon}(f)} \frac{\left|f^{(n)}(w)\right|\left(1-|w|^{2}\right)^{n+\gamma} W(w)}{(1-|w|)^{(p-1)(\beta-1)+\gamma}} d A(w) \\
& \lesssim\left\|f_{1}\right\|_{\mathcal{B}^{\beta, n}}^{p-1}\|f\|_{\mathcal{B}^{\beta, n}} \int_{\Omega_{n, \beta, \epsilon}(f)} \frac{W(w) d A(w)}{\left(1-|w|^{2}\right)^{p \beta-p}} \\
& \lesssim \int_{\Omega_{n, \beta, \epsilon}(f)} \frac{\hat{\omega}(w) d A(w)}{\left(1-|w|^{2}\right)^{p \beta-p+1}}<\infty .
\end{aligned}
$$

Hence, $h \in A_{W}^{p}$. Then, for any $\epsilon>0$, there exists a function $h \in A_{W}^{p} \cap \mathcal{B}^{\beta}$ such that $\| f-$ $h \|_{\mathcal{B}^{\beta, n}} \lesssim \epsilon$, which means that $f \in \mathcal{C}_{\mathcal{B}^{\beta}}\left(A_{\omega}^{p} \cap \mathcal{B}^{\beta}\right)$. The proof is complete.

\section{The operator $C_{\varphi} D^{m}$ on $\mathcal{C}_{\mathcal{B}^{\beta}}\left(A_{\omega}^{p} \cap \mathcal{B}^{\beta}\right)$}

Next, we characterize the boundedness and compactness of the operator $C_{\varphi} D^{m}$ from Bloch type spaces $\mathcal{B}^{\alpha}\left(\mathcal{B}_{0}^{\alpha}\right)$ to $\mathcal{C}_{\mathcal{B}^{\beta}}\left(A_{\omega}^{p} \cap \mathcal{B}^{\beta}\right)$. We denote $\Omega_{n, \beta, \epsilon}(f)$ by $\Omega_{\beta, \epsilon}(f)$ when $n=1$.

Theorem 2 Let $\varphi$ be an analytic self-map of $\mathbb{D}$ and $m \in \mathbb{N}$. Suppose that $0<p<\infty, \omega \in \mathcal{D}$, and $0<\alpha, \beta<\infty$. Then $C_{\varphi} D^{m}: \mathcal{B}^{\alpha} \rightarrow \mathcal{C}_{\mathcal{B}^{\beta}}\left(A_{\omega}^{p} \cap \mathcal{B}^{\beta}\right)$ is bounded if and only if, for any $\epsilon>0$,

$$
\int_{\Gamma_{\epsilon}(\varphi)} \frac{\hat{\omega}(z) d A(z)}{\left(1-|z|^{2}\right)^{p \beta-p+1}}<\infty
$$

where

$$
\Gamma_{\epsilon}(\varphi)=\left\{z \in \mathbb{D}: \frac{\left(1-|z|^{2}\right)^{\beta}}{\left(1-|\varphi(z)|^{2}\right)^{\alpha+m}}\left|\varphi^{\prime}(z)\right| \geq \epsilon\right\} .
$$


Proof Sufficiency. Assume that (2) holds for any $\epsilon>0$. Let $f \in \mathcal{B}^{\alpha}$. Then

$$
\begin{aligned}
\left|\left(C_{\varphi} D^{m} f\right)^{\prime}(z)\right|\left(1-|z|^{2}\right)^{\beta} & =\left|f^{(m+1)}(\varphi(z))\right|\left|\varphi^{\prime}(z)\right|\left(1-|z|^{2}\right)^{\beta} \\
& \leq\|f\|_{\mathcal{B}^{\alpha, m+1}} \frac{\left(1-|z|^{2}\right)^{\beta}}{\left(1-|\varphi(z)|^{2}\right)^{\alpha+m}}\left|\varphi^{\prime}(z)\right| .
\end{aligned}
$$

Thus, for any $\delta>0$, if $\left|\left(C_{\varphi} D^{m} f\right)^{\prime}(z)\right|\left(1-|z|^{2}\right)^{\beta}>\delta$, we have that

$$
\frac{\left(1-|z|^{2}\right)^{\beta}}{\left(1-|\varphi(z)|^{2}\right)^{\alpha+m}}\left|\varphi^{\prime}(z)\right| \geq \frac{\delta}{\|f\|_{\mathcal{B}^{\alpha, m+1}}}=\epsilon
$$

Therefore,

$$
\infty>\int_{\Gamma_{\epsilon}(\varphi)} \frac{\hat{\omega}(z) d A(z)}{\left(1-|z|^{2}\right)^{p \beta-p+1}} \gtrsim \int_{\Omega_{\beta, \delta}\left(C_{\varphi} D^{m} f\right)} \frac{\hat{\omega}(z) d A(z)}{\left(1-|z|^{2}\right)^{p \beta-p+1}}
$$

According to Theorem 1, we get that

$$
C_{\varphi} D^{m} f \in \mathcal{C}_{\mathcal{B}^{\beta}}\left(A_{\omega}^{p} \cap \mathcal{B}^{\beta}\right) .
$$

This means that $C_{\varphi} D^{m} f: \mathcal{B}^{\alpha} \rightarrow \mathcal{C}_{\mathcal{B}^{\beta}}\left(A_{\omega}^{p} \cap \mathcal{B}^{\beta}\right)$ is bounded.

Necessity. Suppose that $C_{\varphi} D^{m} f: \mathcal{B}^{\alpha} \rightarrow \mathcal{C}_{\mathcal{B}^{\beta}}\left(A_{\omega}^{p} \cap \mathcal{B}^{\beta}\right)$ is bounded. It is well known that, for any positive integer $m$, there exist two functions $f_{1}, f_{2} \in \mathcal{B}^{\alpha}$ such that (see [36])

$$
\left|f_{1}^{(m)}(z)\right|+\left|f_{2}^{(m)}(z)\right| \geq \frac{1}{\left(1-|z|^{2}\right)^{\alpha+m-1}} .
$$

Due to our assumption, we get that $f_{1}^{(m)} \circ \varphi, f_{2}^{(m)} \circ \varphi \in \mathcal{C}_{\mathcal{B}^{\beta}}\left(A_{\omega}^{p} \cap \mathcal{B}^{\beta}\right)$. Thus, for any $\epsilon>0$, we have

$$
\int_{\Omega_{\beta, \frac{\epsilon}{2}}\left(f_{1}^{(m)} \circ \varphi\right)} \frac{\hat{\omega}(z) d A(z)}{\left(1-|z|^{2}\right)^{p \beta-p+1}}<\infty
$$

and

$$
\int_{\Omega_{\beta, \frac{\epsilon}{2}}\left(f_{2}^{(m)} \circ \varphi\right)} \frac{\hat{\omega}(z) d A(z)}{\left(1-|z|^{2}\right)^{p \beta-p+1}}<\infty .
$$

If $z \in \Gamma_{\epsilon}(\varphi)$, then we have

$$
\begin{aligned}
& \left(\left|\left(C_{\varphi} D^{m} f_{1}\right)^{\prime}(z)\right|+\left|\left(C_{\varphi} D^{m} f_{2}\right)^{\prime}(z)\right|\right)\left(1-|z|^{2}\right)^{\beta} \\
& \quad=\left(\left|f_{1}^{(m+1)}(\varphi(z))\right|+\left|f_{2}^{(m+1)}(\varphi(z))\right|\right)\left|\varphi^{\prime}(z)\right|\left(1-|z|^{2}\right)^{\beta} \\
& \quad \geq \frac{\left(1-|z|^{2}\right)^{\beta}}{\left(1-|\varphi(z)|^{2}\right)^{\alpha+m}}\left|\varphi^{\prime}(z)\right| \geq \epsilon .
\end{aligned}
$$

This means that, either

$$
\left|\left(C_{\varphi} D^{m} f_{1}\right)^{\prime}(z)\right|\left(1-|z|^{2}\right)^{\beta} \geq \frac{\epsilon}{2}
$$


or

$$
\left|\left(C_{\varphi} D^{m} f_{2}\right)^{\prime}(z)\right|\left(1-|z|^{2}\right)^{\beta} \geq \frac{\epsilon}{2}
$$

Therefore,

$$
\begin{aligned}
\int_{\Gamma_{\epsilon}(\varphi)} \frac{\hat{\omega}(z) d A(z)}{\left(1-|z|^{2}\right)^{p \beta-p+1}} & \leq \int_{\Omega_{\beta, \frac{\epsilon}{2}}\left(f_{1}^{(m)} \circ \varphi\right) \cup \Omega_{\beta, \frac{\epsilon}{2}}\left(f_{2}^{(m)} \circ \varphi\right)} \frac{\hat{\omega}(z) d A(z)}{\left(1-|z|^{2}\right)^{p \beta-p+1}} \\
& \leq \int_{\Omega_{\beta, \frac{\epsilon}{2}}\left(f_{1}^{(m)} \circ \varphi\right)} \frac{\hat{\omega}(z) d A(z)}{\left(1-|z|^{2}\right)^{p \beta-p+1}}+\int_{\left.\Omega_{\beta, \frac{\epsilon}{2}} f_{2}^{(m)} \circ \varphi\right)} \frac{\hat{\omega}(z) d A(z)}{\left(1-|z|^{2}\right)^{p \beta-p+1}} \\
& <\infty .
\end{aligned}
$$

The proof is complete.

Theorem 3 Let $\varphi$ be an analytic self-map of $\mathbb{D}$ and $m \in \mathbb{N}$. Suppose that $0<p<\infty, \omega \in \mathcal{D}$, and $0<\alpha, \beta<\infty$. Then $C_{\varphi} D^{m}: \mathcal{B}_{0}^{\alpha} \rightarrow \mathcal{C}_{\mathcal{B}^{\beta}}\left(A_{\omega}^{p} \cap \mathcal{B}^{\beta}\right)$ is bounded if and only if $\varphi \in \mathcal{C}_{\mathcal{B}^{\beta}}\left(A_{\omega}^{p} \cap\right.$ $\left.\mathcal{B}^{\beta}\right)$ and

$$
\sup _{z \in \mathbb{D}} \frac{\left(1-|z|^{2}\right)^{\beta}}{\left(1-|\varphi(z)|^{2}\right)^{\alpha+m}}\left|\varphi^{\prime}(z)\right|<\infty
$$

Proof The necessity of the conditions can be proved immediately. In fact, we suppose that $C_{\varphi} D^{m}: \mathcal{B}_{0}^{\alpha} \rightarrow \mathcal{C}_{\mathcal{B}^{\beta}}\left(A_{\omega}^{p} \cap \mathcal{B}^{\beta}\right)$ is bounded. Notice that $f_{m}(z)=\frac{z^{m+1}}{(m+1) !} \in \mathcal{B}_{0}^{\alpha}$, then we have

$$
\varphi=C_{\varphi} D^{m} f \in \mathcal{C}_{\mathcal{B}^{\beta}}\left(A_{\omega}^{p} \cap \mathcal{B}^{\beta}\right) .
$$

Since $C_{\varphi} D^{m}: \mathcal{B}_{0}^{\alpha} \rightarrow \mathcal{C}_{\mathcal{B}^{\beta}}\left(A_{\omega}^{p} \cap \mathcal{B}^{\beta}\right)$ is bounded and $\mathcal{C}_{\mathcal{B}^{\beta}}\left(A_{\omega}^{p} \cap \mathcal{B}^{\beta}\right) \subseteq \mathcal{B}^{\beta}$, then $C_{\varphi} D^{m}: \mathcal{B}_{0}^{\alpha} \rightarrow$ $\mathcal{B}^{\beta}$ is bounded. It is easy to see that (3) holds according to [33, Theorem 2.1].

To prove the sufficiency, we assume that $\varphi \in \mathcal{C}_{\mathcal{B}^{\beta}}\left(A_{\omega}^{p} \cap \mathcal{B}^{\beta}\right)$ and

$$
Q:=\sup _{z \in \mathbb{D}} \frac{\left(1-|z|^{2}\right)^{\beta}}{\left(1-|\varphi(z)|^{2}\right)^{\alpha+m}}\left|\varphi^{\prime}(z)\right|<\infty
$$

Let $f \in \mathcal{B}_{0}^{\alpha}$. Then, for any $\epsilon>0$, there is a constant $r(0<r<1)$ such that

$$
\left|f^{(m)}(z)\right|\left(1-|z|^{2}\right)^{\alpha+m-1}<\frac{\epsilon}{Q}, \quad \text { whenever }|z|>r .
$$

Let $z \in \Omega_{\beta, \epsilon}\left(C_{\varphi} D^{m} f\right)$. Then we have

$$
\begin{aligned}
& Q\left|f^{(m+1)}(\varphi(z))\right|\left(1-|\varphi(z)|^{2}\right)^{\alpha+m} \\
& \quad \geq\left|f^{(m+1)}(\varphi(z))\right|\left(1-|\varphi(z)|^{2}\right)^{\alpha+m} \frac{\left(1-|z|^{2}\right)^{\beta}}{\left(1-|\varphi(z)|^{2}\right)^{\alpha+m}}\left|\varphi^{\prime}(z)\right| \\
& \quad=\left|\left(C_{\varphi} D^{m} f\right)^{\prime}(z)\right|\left(1-|z|^{2}\right)^{\beta} \geq \epsilon .
\end{aligned}
$$


This means that $|\varphi(z)| \leq r$. Thus,

$$
\begin{aligned}
& \frac{\|f\|_{\mathcal{B}^{\alpha, m+1}}}{\left(1-r^{2}\right)^{\alpha+m}}\left(1-|z|^{2}\right)^{\beta}\left|\varphi^{\prime}(z)\right| \\
& \quad \geq\left|f^{(m+1)}(\varphi(z))\right|\left(1-|\varphi(z)|^{2}\right)^{\alpha+m} \frac{\left(1-|z|^{2}\right)^{\beta}}{\left(1-|\varphi(z)|^{2}\right)^{\alpha+m}}\left|\varphi^{\prime}(z)\right| \\
& \quad=\left|\left(C_{\varphi} D^{m} f\right)^{\prime}(z)\right|\left(1-|z|^{2}\right)^{\beta} \geq \epsilon .
\end{aligned}
$$

Let $\delta=\frac{\left(1-r^{2}\right)^{\alpha+m} \epsilon}{\|f\|_{\mathcal{B}^{\alpha, m+1}}}$. Then $\left|\varphi^{\prime}(z)\right|\left(1-|z|^{2}\right)^{\beta} \geq \delta$. Hence, $\Omega_{\beta, \epsilon}\left(C_{\varphi} D^{m} f\right) \subseteq \Omega_{\beta, \delta}(\varphi)$. Due to $\varphi \in$ $\mathcal{C}_{\mathcal{B}^{\beta}}\left(A_{\omega}^{p} \cap \mathcal{B}^{\beta}\right)$, we obtain

$$
\infty>\int_{\Omega_{\beta, \delta}(\varphi)} \frac{\hat{\omega}(z) d A(z)}{\left(1-|z|^{2}\right)^{p \beta-p+1}} \geq \int_{\Omega_{\beta, \epsilon}\left(C_{\varphi} D^{m} f\right)} \frac{\hat{\omega}(z) d A(z)}{\left(1-|z|^{2}\right)^{p \beta-p+1}}
$$

According to Theorem 1, we get that $C_{\varphi} D^{m} f \in \mathcal{C}_{\mathcal{B}^{\beta}}\left(A_{\omega}^{p} \cap \mathcal{B}^{\beta}\right)$. Therefore, $C_{\varphi} D^{m}: \mathcal{B}_{0}^{\alpha} \rightarrow$ $\mathcal{C}_{\mathcal{B}^{\beta}}\left(A_{\omega}^{p} \cap \mathcal{B}^{\beta}\right)$ is bounded. The proof is complete.

Theorem 4 Let $\varphi$ be an analytic self-map of $\mathbb{D}$ and $m \in \mathbb{N}$. Suppose that $0<p<\infty, \omega \in \mathcal{D}$, and $0<\alpha, \beta<\infty$. Then the following statements are equivalent:

(i) $C_{\varphi} D^{m}: \mathcal{B}^{\alpha} \rightarrow \mathcal{C}_{\mathcal{B}^{\beta}}\left(A_{\omega}^{p} \cap \mathcal{B}^{\beta}\right)$ is compact;

(ii) $C_{\varphi} D^{m}: \mathcal{B}_{0}^{\alpha} \rightarrow \mathcal{C}_{\mathcal{B}^{\beta}}\left(A_{\omega}^{p} \cap \mathcal{B}^{\beta}\right)$ is compact;

(iii) $\varphi \in \mathcal{C}_{\mathcal{B}^{\beta}}\left(A_{\omega}^{p} \cap \mathcal{B}^{\beta}\right)$ and

$$
\lim _{|\varphi(z)| \rightarrow 1} \frac{\left(1-|z|^{2}\right)^{\beta}}{\left(1-|\varphi(z)|^{2}\right)^{\alpha+m}}\left|\varphi^{\prime}(z)\right|=0 .
$$

Proof (i) $\Rightarrow$ (ii). The implication is obvious because $\mathcal{B}_{0}^{\alpha} \subseteq \mathcal{B}^{\alpha}$.

(ii) $\Rightarrow$ (iii). Assume that $C_{\varphi} D^{m}: \mathcal{B}_{0}^{\alpha} \rightarrow \mathcal{C}_{\mathcal{B}^{\beta}}\left(A_{\omega}^{p} \cap \mathcal{B}^{\beta}\right)$ is compact. Obviously, $C_{\varphi} D^{m}$ : $\mathcal{B}_{0}^{\alpha} \rightarrow \mathcal{C}_{\mathcal{B}^{\beta}}\left(A_{\omega}^{p} \cap \mathcal{B}^{\beta}\right)$ is bounded. According to Theorem 3 , we obtain $\varphi \in \mathcal{C}_{\mathcal{B}^{\beta}}\left(A_{\omega}^{p} \cap \mathcal{B}^{\beta}\right)$. On the other hand, it is obvious that $\mathcal{C}_{\mathcal{B}^{\beta}}\left(A_{\omega}^{p} \cap \mathcal{B}^{\beta}\right) \subseteq \mathcal{B}^{\beta}$. Then $C_{\varphi} D^{m}: \mathcal{B}_{0}^{\alpha} \rightarrow \mathcal{B}^{\beta}$ is compact. This clearly implies that (4) holds by [33, Theorem 2.2].

(iii) $\Rightarrow$ (i). According to the assumed condition, we see that there exists $r(0<r<1)$ such that

$$
\frac{\left(1-|z|^{2}\right)^{\beta}}{\left(1-|\varphi(z)|^{2}\right)^{\alpha+m}}\left|\varphi^{\prime}(z)\right|<\frac{\epsilon}{2}, \quad \text { whenever }|\varphi(z)|>r
$$

Let $z \in \Gamma_{\epsilon}(\varphi)$, then $|\varphi(z)| \leq r$. Therefore,

$$
\frac{\left(1-|z|^{2}\right)^{\beta}}{\left(1-r^{2}\right)^{\alpha+m}}\left|\varphi^{\prime}(z)\right| \geq \frac{\left(1-|z|^{2}\right)^{\beta}}{\left(1-|\varphi(z)|^{2}\right)^{\alpha+m}}\left|\varphi^{\prime}(z)\right| \geq \epsilon .
$$

Thus

$$
\left|\varphi^{\prime}(z)\right|\left(1-|z|^{2}\right)^{\beta} \geq\left(1-r^{2}\right)^{\alpha+m} \epsilon .
$$

Set $\delta=\left(1-r^{2}\right)^{\alpha+m} \epsilon$. Then $z \in \Omega_{\beta, \delta}(\varphi)$. Since $\varphi \in \mathcal{C}_{\mathcal{B}^{\beta}}\left(A_{\omega}^{p} \cap \mathcal{B}^{\beta}\right)$, we have

$$
\infty>\int_{\Omega_{\beta, \delta}(\varphi)} \frac{\hat{\omega}(z) d A(z)}{\left(1-|z|^{2}\right)^{p \beta-p+1}} \gtrsim \int_{\Gamma_{\epsilon}(\varphi)} \frac{\hat{\omega}(z) d A(z)}{\left(1-|z|^{2}\right)^{p \beta-p+1}} .
$$


According to Theorem 2, $C_{\varphi} D^{m}: \mathcal{B}^{\alpha} \rightarrow \mathcal{C}_{\mathcal{B}^{\beta}}\left(A_{\omega}^{p} \cap \mathcal{B}^{\beta}\right)$ is bounded. We know that $C_{\varphi} D^{m}$ : $\mathcal{B}^{\alpha} \rightarrow \mathcal{B}^{\beta}$ is compact by [33, Theorem 2.2]. Therefore, $C_{\varphi} D^{m}: \mathcal{B}^{\alpha} \rightarrow \mathcal{C}_{\mathcal{B}^{\beta}}\left(A_{\omega}^{p} \cap \mathcal{B}^{\beta}\right)$ is compact. The proof is complete.

Theorem 5 Let $\varphi$ be an analytic self-map of $\mathbb{D}$ and $m \in \mathbb{N}$. Suppose that $0<p<\infty, \omega \in \mathcal{D}$, and $0<\beta<\infty$. Then $C_{\varphi} D^{m}: \mathcal{C}_{\mathcal{B}^{\beta}}\left(A_{\omega}^{p} \cap \mathcal{B}^{\beta}\right) \rightarrow \mathcal{C}_{\mathcal{B}^{\beta}}\left(A_{\omega}^{p} \cap \mathcal{B}^{\beta}\right)$ is compact if and only if $\varphi \in \mathcal{C}_{\mathcal{B}^{\beta}}\left(A_{\omega}^{p} \cap \mathcal{B}^{\beta}\right)$ and

$$
\lim _{|\varphi(z)| \rightarrow 1} \frac{\left(1-|z|^{2}\right)^{\beta}}{\left(1-|\varphi(z)|^{2}\right)^{\beta+m}}\left|\varphi^{\prime}(z)\right|=0 .
$$

Proof Assume that $C_{\varphi} D^{m}: \mathcal{C}_{\mathcal{B}^{\beta}}\left(A_{\omega}^{p} \cap \mathcal{B}^{\beta}\right) \rightarrow \mathcal{C}_{\mathcal{B}^{\beta}}\left(A_{\omega}^{p} \cap \mathcal{B}^{\beta}\right)$ is compact. Thus $C_{\varphi} D^{m}$ : $\mathcal{C}_{\mathcal{B}^{\beta}}\left(A_{\omega}^{p} \cap \mathcal{B}^{\beta}\right) \rightarrow \mathcal{C}_{\mathcal{B}^{\beta}}\left(A_{\omega}^{p} \cap \mathcal{B}^{\beta}\right)$ is bounded. So we obtain $\varphi \in \mathcal{C}_{\mathcal{B}^{\beta}}\left(A_{\omega}^{p} \cap \mathcal{B}^{\beta}\right)$ since $\frac{z^{m+1}}{(m+1) !} \in$ $\mathcal{C}_{\mathcal{B}^{\beta}}\left(A_{\omega}^{p} \cap \mathcal{B}^{\beta}\right)$. It is well known that $\mathcal{B}_{0}^{\beta}$ is the closure of all polynomials in $\mathcal{B}^{\beta}$ and the space $A_{\omega}^{p}$ contains all polynomials. Therefore, $C_{\varphi} D^{m}: \mathcal{B}_{0}^{\beta} \rightarrow \mathcal{C}_{\mathcal{B}^{\beta}}\left(A_{\omega}^{p} \cap \mathcal{B}^{\beta}\right)$ is compact. According to Theorem 4, we see that (5) holds.

Conversely, suppose that $\varphi \in \mathcal{C}_{\mathcal{B}^{\beta}}\left(A_{\omega}^{p} \cap \mathcal{B}^{\beta}\right)$ and (5) holds. By [33, Theorem 2.2], we see that $C_{\varphi} D^{m}: \mathcal{B}^{\beta} \rightarrow \mathcal{B}^{\beta}$ is compact. By Theorem 4, we know that $C_{\varphi} D^{m}: \mathcal{B}^{\beta} \rightarrow \mathcal{C}_{\mathcal{B}^{\beta}}\left(A_{\omega}^{p} \cap\right.$ $\left.\mathcal{B}^{\beta}\right)$ is compact. Since $\mathcal{C}_{\mathcal{B}^{\beta}}\left(A_{\omega}^{p} \cap \mathcal{B}^{\beta}\right) \subseteq \mathcal{B}^{\beta}$, we obtain $C_{\varphi} D^{m}: \mathcal{C}_{\mathcal{B}^{\beta}}\left(A_{\omega}^{p} \cap \mathcal{B}^{\beta}\right) \rightarrow \mathcal{C}_{\mathcal{B}^{\beta}}\left(A_{\omega}^{p} \cap\right.$ $\left.\mathcal{B}^{\beta}\right)$ is compact. The proof is complete.

\section{Acknowledgements}

The author thanks the referees for their numerous helpful suggestions.

\section{Funding}

This project was partially supported by the Macao Science and Technology Development Fund (No. 186/2017/A3).

\section{Availability of data and materials}

Not applicable.

\section{Competing interests}

The author declares that he has no competing interests.

\section{Authors' contributions}

The author has contributed to the writing of this paper. He read and approved the manuscript.

\section{Publisher's Note}

Springer Nature remains neutral with regard to jurisdictional claims in published maps and institutional affiliations.

Received: 7 May 2019 Accepted: 26 November 2019 Published online: 11 December 2019

\section{References}

1. Anderson, J.: Bloch Functions: The Basic Theory, Operators and Function Theory. NATO Adv. Sci. Inst. Ser. C Math. Phys. Sci., vol. 153, pp. 1-17. Reidel, Dordrechet (1985). https://doi.org/10.1007/978-94-009-5374-1

2. Anderson, J., Clunie, J., Pommerenke, C.: On Bloch functions and normal functions. J. Reine Angew. Math. 270, 12-37 (1974)

3. Aulaskari, R., Zhao, R.: Composition operators and closures of some Möbius invariant spaces in the Bloch space. Math. Scand. 107, 139-149 (2010). https://doi.org/10.7146/math.scand.a-15147

4. Bao, G., Göğüşs, N.: On the closures of Dirichlet type spaces in the Bloch space. Complex Anal. Oper. Theory 13, 45-59 (2019). https://doi.org/10.1007/s11785-017-0676-2

5. Cowen, C.C., MacCluer, B.D.: Composition Operators on Spaces of Analytic Functions. CRC Press, Boca Raton (1995)

6. Duren, P.: Theory of HP Spaces. Academic Press, New York (1970)

7. Galán, N.M., Nicolau, A.: The closure of the Hardy space in the Bloch norm. Algebra Anal. 22, 75-81 (2010). Translation in St. Petersburg Math. J. 22, 55-59 (2011). https://doi.org/10.1090/S1061-0022-2010-01132-4

8. Galanopoulos, P., Galán, N.M., Pau, J.: Closure of Hardy spaces in the Bloch space. J. Math. Anal. Appl. 429, 1214-1221 (2015). https://doi.org/10.1016/j.jmaa.2015.04.075

9. Galanopoulos, P., Girela, D.: The closure of Dirichlet spaces in the Bloch space. Ann. Acad. Sci. Fenn. 44, 91-101 (2019). https://doi.org/10.5186/aasfm.2019.4402

10. Ghatage, P., Zheng, D.: Analytic functions of bounded mean oscillation and the Bloch space. Integral Equ. Oper. Theory 17, 501-515 (1993). https://doi.org/10.1007/BF01200391 
11. $\mathrm{Hu}, \mathrm{N}$. , Zhu, X.: Composition operators and the closure of Morrey space in the Bloch space. J. Funct. Spaces 2019, Article ID 2834865 (2019). https://doi.org/10.1155/2019/2834865

12. Li, S.: Differences of generalized composition operators on the Bloch space. J. Math. Anal. Appl. 394, 706-711 (2012). https://doi.org/10.1016/j.jmaa.2012.04.009

13. Li, S., Stević, S.: Composition followed by differentiation between Bloch type spaces. J. Comput. Anal. Appl. 9(2), 195-205 (2007)

14. Li, S., Stević, S.: Composition followed by differentiation from mixed-norm spaces to $\alpha$-Bloch spaces. Mat. Sb. 199, 117-128 (2008). https://doi.org/10.1070/SM2008v199n12ABEH003983

15. Li, S., Stević, S.: Generalized composition operators on Zygmund spaces and Bloch type spaces. J. Math. Anal. Appl. 338, 1282-1295 (2008). https://doi.org/10.1016/j.jmaa.2007.06.013

16. Li, S., Stević, S.: Products of composition and integral type operators from $H^{\infty}$ to the Bloch space. Complex Var. Elliptic Equ. 53(5), 463-474 (2008). https://doi.org/10.1080/17476930701754118

17. Li, S., Stević, S.: Composition followed by differentiation between $H^{\infty}$ and $\alpha$-Bloch spaces. Houst. J. Math. 35 $327-340(2009)$

18. Liu, X., Li, S.: Norm and essential norm of a weighted composition operator on the Bloch space. Integral Equ. Oper. Theory 87, 309-325 (2017). https://doi.org/10.1007/s00020-017-2349-y

19. Lou, Z: Composition operators on Bloch type spaces. Analysis 23, 81-95 (2003). https://doi.org/10.1524/anly.2003.23.1.81

20. Lou, Z., Chen, W.: Distances from Bloch functions to $\mathcal{Q}_{K}$-type space. Integral Equ. Oper. Theory 67, 171-181 (2010). https://doi.org/10.1007/s00020-010-1762-2

21. Madigan, K., Matheson, A.: Compact composition operators on the Bloch space. Trans. Am. Math. Soc. 347, 2679-2687 (1995). https://doi.org/10.2307/2154848

22. Peláez, J., Rättyä, J.: Weighted Bergman spaces induced by rapidly increasing weights. Mem. Am. Math. Soc. 227, 1066 (2014)

23. Peláez, J., Rättyä, J.: Embedding theorems for Bergman spaces via harmonic analysis. Math. Ann. 362, 205-239 (2015). https://doi.org/10.1007/s00208-014-1108-5

24. Peláez, J., Rättyä, J.: Bergman projection induced by radial weight. arXiv:1902.09837

25. Peláez, J., Rättyä, J., Sierra, K.: Berezin transform and Toeplitz operators on Bergman spaces induced by regular weights. J. Geom. Anal. 28, 656-687 (2018). https://doi.org/10.1007/s12220-017-9837-9

26. Qian, R., Li, S.: Composition operators and closures of Dirichlet type spaces $\mathcal{D}_{\alpha}$ in the logarithmic Bloch space. Indag. Math. 29, 1432-1440 (2018). https://doi.org/10.1016/j.indag.2018.07.006

27. Qian, R., Li, S.: Composition operators and closures of Dirichlet type spaces $\mathcal{D}_{\mu}$ in Bloch type spaces. Anal. Math 45(1), 121-132 (2019). https://doi.org/10.1007/s10476-018-0406-7

28. Tjani, M.: Compact composition operators on some Möbius invariant Banach space. PhD dissertation, Michigan State University (1996)

29. Tjani, M.: Distance of a Bloch function to the little Bloch space. Bull. Aust. Math. Soc. 74, 101-119 (2006). https://doi.org/10.1017/S0004972700047493

30. Wulan, H., Zheng, D., Zhu, K.: Compact composition operators on BMOA and the Bloch space. Proc. Am. Math. Soc. 137, 3861-3868 (2009). https://doi.org/10.1090/S0002-9939-09-09961-4

31. Zhao, R.: Distances from Bloch functions to some Möbius invariant spaces. Ann. Acad. Sci. Fenn., Math. 33, 303-313 (2008)

32. Zhao, R.: Essential norms of composition operators between Bloch type spaces. Proc. Am. Math. Soc. 138, 2537-2546 (2010). https://doi.org/10.1090/50002-9939-10-10285-8

33. Zhou, J., Zhu, X.: Product of differentiation and composition operators on Bloch type spaces. Publ. Inst. Math. 99(113), 295-300 (2016). https://doi.org/10.2298/PIM150203021Z

34. Zhu, K.: Bloch type spaces of analytic functions. Rocky Mt. J. Math. 23, 1143-1177 (1993). https://doi.org/10.1216/rmjm/1181072549

35. Zhu, K:: Operator Theory in Function Spaces. Am. Math. Soc., Providence (2007)

36. Zhu, X: Generalized weighted composition operators from Bloch type spaces to weighted Bergman spaces. Indian J. Math. 49, 139-150 (2007). https://doi.org/10.4134/JKMS.2009.46.6.1219

37. Zhu, X:: Essential norm and compactness of the product of differentiation and composition operators on Bloch type spaces. Math. Inequal. Appl. 19, 325-334 (2016). https://doi.org/10.7153/mia-19-24

\section{Submit your manuscript to a SpringerOpen ${ }^{\circ}$ journal and benefit from:}

- Convenient online submission

- Rigorous peer review

- Open access: articles freely available online

- High visibility within the field

- Retaining the copyright to your article

Submit your next manuscript at $\gg$ springeropen.com 